\title{
Definición de las condiciones de temperatura y almacenamiento adecuadas en la detección de ADN de Leishmania por PCR en flebotominos
}

\author{
Olga L. Cabrera ${ }^{1}$, Leonard E. Munstermann ${ }^{2}$, Rocío Cárdenas ${ }^{1}$, Reynaldo Gutiérrez ${ }^{3}$, Cristina Ferro ${ }^{1}$ \\ ${ }^{1}$ Laboratorio de Entomología, Instituto Nacional de Salud, Bogotá, D.C., Colombia. \\ 2 Departamento de Epidemiología y Salud Publica, Escuela de Medicina, Yale University, New Haven, \\ EE.UU. \\ ${ }^{3}$ Centro de Investigaciones en Enfermedades Tropicales, CINTROP, Universidad Industrial de Santander, \\ Bucaramanga, Colombia.
}

Para estudios epidemiológicos y programas de control de las leishmaniasis es importante la determinación taxonómica del insecto vector y del agente etiológico causante de esta enfermedad. Por su eficacia y sensibilidad, la utilización de técnicas moleculares, como la reacción en cadena de la polimerasa (PCR) ha permitido avances en entomología médica. La metodología tradicional usada para la búsqueda de infección en los flebótomos es un método dispendioso que requiere mucho tiempo y capacitación para emitir un diagnóstico acertado. En el presente trabajo se evaluaron las condiciones y prácticas adecuadas para el almacenamiento de flebótomos con el propósito de aplicar la PCR. Hembras de Lutzomyia longipalpis de la colonia del Instituto Nacional de Salud se infectaron experimentalmente con una cepa de Leishmania chagasi del valle alto del río Magdalena (Quipile, Cundinamarca). Los insectos infectados se preservaron en tres soluciones: etanol al $100 \%$, etanol al $70 \%$ y en buffer Tris$\operatorname{EDTA}(\mathrm{TE})$; submuestras de cada una se almacenaron $\mathrm{a}-80^{\circ} \mathrm{C},-20^{\circ} \mathrm{C}$ y a temperatura ambiente. Para determinar los porcentajes de infección, algunas de las hembras se disecaron para buscar las formas flageladas al microscopio. La extracción del ${ }_{K} A D N$ se realizó con Chelex 100 . Para la amplificación se utilizaron los iniciadores OL1 y OL2 de Leishmania con electroforesis en geles de agarosa al 1\%. En cada una de las condiciones descritas se logró la amplificación de un fragmento de $\approx 120 \mathrm{pb}$, correspondiente a Leishmania spp. Estos resultados muestran la ventaja de incorporar como técnica de rutina la PCR para detectar flebótomos infectados de zonas endémicas de leishmaniasis visceral. Por costo-efectividad, se concluyó que las muestras entomológicas para estudios de incriminación vectorial utilizando la PCR, se pueden preservar a temperatura ambiente en etanol al $70 \%$.

Palabras clave: Lutzomyia, Leishmania, preservación, PCR, Chelex 100.

\section{Definition of temperature and storage adequate conditions in Leishmania DNA detection by PCR in phlebotomine samples}

For epidemiological studies and control programs of leishmaniasis, taxonomic identification of the etiologic agent of the disease in the insect vector is of critical importance. The implementation of molecular techniques such as the polymerase chain reaction (PCR) has permitted great advances in the efficacy and sensitivity of parasite identification. Previously, these investigations involved labor-intensive dissections and required expert personnel. The present work evaluates the effects of storage methods of phlebotomine samples in the optimization of PCR identification of Leishmania. Females of Lutzomyia longipalpis, from the colony of the Instituto Nacional de Salud, were experimentally infected with Leishmania chagasi (=L. infantum), from the upper Magdalena Valley (Quipile, Cundinamarca, Colombia). The infected insects were preserved in three solutions: $100 \%$ ethanol, $70 \%$ ethanol, and TE; subsamples of each class were stored at $-80^{\circ} \mathrm{C},-20^{\circ} \mathrm{C}$ and room temperature. To determine infection rates, samples were dissected and screened microscopically. Chelex 100 was used for extraction of total Leishmania DNA. For PCR amplification, the kinetoplastic minicircle DNA primers OL1 and OL2 of Leishmania were used, and the products were visualized by electrophoresis in $1 \%$ agarose gels. For each of the 
3 storage conditions, amplifications were successful, producing $\mathrm{a} \approx 120$ base pair product unique to Leishmania. The results demonstrated the advantage of PCR as a routine screening method for detecting infected flies in endemic foci of visceral leishmaniasis. Since storage method did not affect PCR amplification success, the most cost effective method $-70 \%$ ethanol at room temperature- is the option recommended for storing entomological samples in vector incrimination studies.

Key words: Sand fly, Leishmania, preservation, PCR, Chelex 100.

La leishmaniasis visceral es una enfermedad especialmente importante en salud pública por su asociación con mortalidad infantil en ausencia de tratamiento. En Latinoamérica la causan parásitos Leishmania infantum (=L. chagasi) y se transmite a los humanos por la picadura de pequeños dípteros hematófagos de la familia Psychodidae. Las especies del complejo Lutzomyia longipalpis son consideradas los vectores más importantes (1-3). Se encuentran distribuidas en la mayoría de los focos endémicos de la enfermedad en Centro y Suramérica (4). Como vector alterno del parásito, en algunos países se ha señalado a Lutzomyia evansi. En Colombia, el comportamiento de $L$. evansi como vector de $L$. chagasi se demostró en San Andrés de Sotavento, Córdoba (5). En Costa Rica se ha registrado como la segunda especie más abundante después de L. longipalpis (6). En Venezuela, en un estudio llevado a cabo en Guayavita, Aragua, L. evansi fue la especie más abundante y se encontró naturalmente infectada con Leishmania spp. (7). En localidades donde estas dos especies, $L$. longipalpis y $L$. evansi, presentan distribución simpátrica, se ha sugerido una alternancia estacional entre ellas, ya que los picos de abundancia máxima de estas poblaciones no son simultáneos, por efecto de las variables climáticas en las poblaciones de estos insectos, especial-mente en las de L. longipalpis.

En estudios de incriminación vectorial, la presencia del parásito en el insecto es uno de los cuatro criterios propuestos por Killick-Kendrick como indispensables para considerar un flebótomo como vector de Leishmania (8). La metodología ampliamente usada para la búsqueda de infección en los flebótomos, por lo menos hasta la década

Correspondencia:

Cristina Ferro, mferro@ins.gov.co

Recibido: 04/19/02; aceptado: 27/08/02 pasada, se basaba en el hallazgo de las formas flageladas en el intestino de los probables vectores. Este es un método dispendioso que requiere mucho tiempo y capacitación para emitir un diagnóstico acertado $(9,10)$.

La incorporación de técnicas de biología molecular, calificadas como altamente sensibles y relativamente rápidas, ha permitido diferenciar entre especies y subespecies del género Leishmania en el flebótomo, utilizando pocas cantidades de ADN mitocondrial. La PCR como una de estas técnicas con capacidad de producir cientos de copias de ADN se ha utilizado en estudios entomológicos con diferentes fines $(11,12)$.

Con relación a los medios de almacenamiento para la preservación de las muestras entomológicas a partir de las cuales se obtiene el ADN blanco para aplicación de pruebas moleculares, en 1998 Uezato et al. (13) evaluaron tres formas diferentes de preservación de biopsias de piel humana para determinar por PCR las tasas de infección con Leishmania. Parte de las muestras fueron congeladas, otras embebidas en formol y una tercera parte en etanol al $100 \%$. Concluyeron que para el diagnóstico de leishmaniasis por PCR, la preservación de las muestras en etanol al $100 \%$ es adecuada tanto para su transporte como para su almacenamiento.

En cuanto a la temperatura en la cual se mantienen las muestras para diagnóstico por PCR, se han señalado especialmente las siguientes: temperatura ambiente, $-20^{\circ} \mathrm{C}$ y $-70{ }^{\circ} \mathrm{C}$ (14-16).

Para la extracción del ADN, el método fenolcloroformo se describe con más frecuencia (1719). Sin embargo, para la obtención del ADN de protozoarios a partir de biopsias de mamíferos y de intestinos infectados de mosquitos del género Anopheles, existen otros métodos que se han 
usado con buenos resultados, como la cromatografía de intercambio iónico (15) y extracción con Chelex 100 (20). En los últimos años, el Laboratorio de Entomología del Instituto Nacional de Salud estandarizó la técnica de extracción con Chelex 100 al $5 \%$ del ADN de Leishmania presente en el intestino de insectos del género Lutzomyia (21).

En el presente trabajo se evaluaron las condiciones de temperatura y almacenamiento adecuados para detectar el ADN de Leishmania en el vector por la técnica de PCR. Hembras de Lutzomyia longipalpis infectadas experimentalmente se preservaron en: etanol al $100 \%$, etanol al $70 \%$ y buffer Tris-EDTA (TE), bajo las siguientes temperaturas: $-80{ }^{\circ} \mathrm{C},-20{ }^{\circ} \mathrm{C}$ y temperatura ambiente. Se concluyó que todas las condiciones evaluadas son adecuadas. Por costos, facilidad de adquisición y manejo de las muestras en condiciones de campo, se recomienda utilizar etanol al $70 \%$ a temperatura ambiente para estudios de incriminación de vectores de leishmaniasis.

\section{Materiales y métodos}

\section{Infección de Lutzomyia longipalpis con Leishmania chagasi}

Hembras adultas de una colonia de L. longipalpis de tres días de nacidas fueron alimentadas con una mezcla de promastigotes de $L$. chagasi y glóbulos rojos lavados en una proporción $2: 1$, a través de membrana de pollito de dos días de edad. El volumen final de esta mezcla fue de 3 $\mathrm{ml}$ y la cantidad ingerida por insecto fue de $0,5 \mu \mathrm{l}$ aproximadamente (22). Los parásitos y los insectos provenían originalmente del foco endémico de leishmaniasis visceral ubicado en el valle alto del río Magdalena en el departamento de Cundinamarca; la cepa de Leishmania, del municipio de Quipile (4 44' 03" LN, 74 33' 47" LO), y los flebótomos de la vereda El Callejón en el municipio de Ricaurte ( $4^{\circ} 18^{\prime} \mathrm{LN}, 74^{\circ} 42^{\prime} \mathrm{LO}$ ) (23). Las hembras alimentadas, es decir, aquéllas en las que se podía observar la ingestión de sangre, se pasaron a una jaula de muselina de $20 \times 20 \mathrm{~cm}$, a una temperatura entre los 22 y 25 ${ }^{\circ} \mathrm{C}$. La jaula con las hembras se introdujo en una bolsa de plástico para mantener la humedad relativa $(70 \%)$. Como fuente de carbohidrato, se ofreció diariamente a las hembras una solución azucarada (saturada) embebida en motas de algodón colocadas en la parte superior de la jaula. La presencia de flagelados se confirmó por observación directa con ayuda de un microscopio de luz a 400X, el día siete postalimentación. Los intestinos positivos y negativos se preservaron para los ensayos de PCR.

Entre ocho y doce días después de la comida infectiva, el resto de las hembras se retiró de las jaulas y se preservaron individualmente en los tubos de reacción, en tres soluciones diferentes: etanol al 100\% (41 muestras), etanol al 70\% (51 muestras) y buffer Tris EDTA (TE pH 8) (93 muestras). Submuestras de las muestras anteriores, en cada una de las soluciones, se preservaron a $-80{ }^{\circ} \mathrm{C},-20{ }^{\circ} \mathrm{C}$ y temperatura ambiente.

\section{Extracción del ADN}

Uno o dos días antes de la extracción, las hembras individualizadas y sin disecar, se trituraron manualmente para romper los tejidos. Con ayuda de una varilla de vidrio muy fina $(0,1$ $\mathrm{cm}$ ), cada hembra se molió en el mismo tubo, en el medio de almacenamiento en el que fueron preservadas. Después del proceso, los tubos con las muestras se colocaron nuevamente a la temperatura correspondiente. El protocolo para la obtención del ADN fue el siguiente: 1) centrifugación durante 3 minutos a 10.000 rpm; 2) eliminación del sobrenadante; 3) adición de $100 \mu \mathrm{l}$ de Chelex 100 al 5\%; 4) incubación de las muestras a $56{ }^{\circ} \mathrm{C}$ durante 30 minutos en baño María; 5) agitación en vórtex durante 10 segundos; 6) ebullición durante 8 minutos; 7) agitación en vórtex durante 10 segundos; 8) centrifugación durante 3 minutos a $10.000 \mathrm{rpm}$, y 9) obtención del ADN del sobrenadante como plantilla para la PCR.

\section{Amplificación por PCR del ADN mitocondrial}

La amplificación se realizó utilizando como iniciadores un par de oligonucleótidos universales para Leishmania diseñados sobre un área de la región conservada de los minicírculos del ${ }_{K} A D N$, OL1: GGG GAG GGG CGT TCT GCG AA (16) y OL2: CCG CCC CTA TTT TAC ACC AAC CCC 
$(15,24,25)$, que produce una banda de $\approx 120$ pares de bases. EL volumen final por tubo de reacción fue de $50 \mu \mathrm{l}$, de los cuales $4 \mu \mathrm{l}$ correspondían a la plantilla de ADN de cada una de las muestras y $46 \mu$ l de la mezcla de reacción, que contenía $400 \mathrm{ng}$ de cada iniciador, 2,0 mM de $\mathrm{MgCl}_{2}, 0,05 \mathrm{mM}$ de dNTPs, 0,05 U de Taq polimerasa y el buffer (Promega) $1 \mathrm{X}$, el cual contiene $10 \mathrm{mM}$ de Tris- $\mathrm{HCl}\left(\mathrm{pH} \mathrm{9,0}\right.$ a $\left.25^{\circ} \mathrm{C}\right), 50$ $\mathrm{mM} \mathrm{KCl} \mathrm{y} 0,1 \%$ de Triton R X-100.

El proceso de amplificación se realizó en un termociclador Perkin-Elmer 2.400 bajo las siguientes condiciones: predenaturación durante 4 minutos a $94{ }^{\circ} \mathrm{C}$, seguido por 30 ciclos de denaturación a $94{ }^{\circ} \mathrm{C}$ durante 30 segundos, anillaje a $55^{\circ} \mathrm{C}$ durante 30 segundos y extensión a $72{ }^{\circ} \mathrm{C}$ durante 30 segundos, con una extensión adicional de 10 minutos a $72{ }^{\circ} \mathrm{C}$. Como control de reacción se utilizó la misma cepa de $L$. chagasi suministrada a los insectos en el proceso de infección y como control negativo se preparó el día de la extracción un intestino de L. longipalpis de colonia con 48 horas de nacido, siguiendo el mismo procedimiento descrito para las muestras.

Del producto amplificado se tomaron $12 \mu \mathrm{l}$ y se mezclaron con $4 \mu \mathrm{l}$ de buffer de carga, el cual contenía azul de bromofenol al 0,1\%. La electroforesis se realizó en geles de agarosa al $1 \%$, teñidos con bromuro de etidio y visualizados con luz UV. Como marcador de peso molecular se empleó el fago x174 digerido con Hae III.

\section{Resultados}

De las 185 hembras alimentadas con la mezcla parásito y sangre, se disecaron $40(21,6 \%)$ y los intestinos se observaron directamente al microscopio de luz, registrando un porcentaje de positividad superior al $95 \%$. El resultado de la PCR fue negativo para los intestinos señalados como negativos por observación directa $(3,5 \%)$ y positivo para los intestinos infectados con promastigotes $(96,5 \%)$.

Una submuestra de 21 hembras de las 145 alimentadas y no disecadas se incluyeron en los ensayos de PCR. La positividad fue del $81 \%$.

Usando la reacción en cadena de la polimerasa se obtuvo la banda esperada a partir de material preservado en etanol al $100 \%$, etanol al $70 \%$ y en buffer Tris EDTA (TE, pH 8). Se observaron únicamente bandas de $\approx 120$ pares de bases correspondientes al fragmento de la región conservada del ${ }_{\mathrm{K}} \mathrm{ADN}$ de Leishmania.

La obtención del ADN por el método descrito resultó adecuada y eficiente, permitiendo la visualización de la banda esperada en geles de agarosa después de 30 ciclos de amplificación. Todos los productos mostraron una intensidad uniforme sugiriendo una concentración similar en estas muestras. La figura 1 permite observar productos amplificados de las muestras almacenadas en los tres medios a temperatura ambiente. La figura 2 enseña los productos de las muestras de ejemplares completos, almacenados en cada uno de los medios y bajo las tres temperaturas establecidas.

\section{Discusión}

Actualmente, la detección de parásitos del género Leishmania en los insectos incluye métodos que van desde la búsqueda directa de los flagelados en el tracto digestivo del insecto hasta técnicas moleculares que usan principalmente ${ }_{\mathrm{K}} \mathrm{ADN}$ (12,26-30). La técnica de reacción en cadena de la polimerasa (PCR), por su alta sensibilidad y especificidad, ha facilitado adelantar estudios ecoepidemiológicos en áreas de alta endemicidad, suministrando datos más precisos en periodos de tiempo más cortos $(17,29)$. Aunque la correcta amplificación del ADN depende especialmente de la apropiada selección de los iniciadores, en los estudios de incriminación vectorial, el método de almacenamiento de la muestra y el proceso de extracción son dos aspectos importantes que contribuyen al éxito de esta amplificación. Lo anterior teniendo en cuenta que, además del ADN del parásito, existe la posibilidad de contar con la presencia de ADN de tejidos del insecto, de otros parásitos o de microorganismos que pueden inhibir o interferir en la reacción.

En el presente estudio se evaluó la PCR como una técnica útil en la detección de parásitos de Leishmania presentes en el insecto vector. La incorporación del uso del Chelex 100 en el proceso de extracción de ADN de protozoarios a partir de muestras de pequeños insectos es una 
alternativa práctica y eficiente frente a otros protocolos (31). La experiencia de Schriefer et al. (20) es diferente a la nuestra respecto a la extracción de ADN con Chelex 100, considerando que ellos tuvieron algunas dificultades para detectar infección con parásitos de malaria tanto en mosquitos individuales como en pequeños volúmenes de sangre. En nuestro ensayo, el ADN de Leishmania en flebótomos infectados experimentalmente se pudo visualizar sin ningún problema (figura 1).

En cuanto a los medios de almacenamiento a diferentes temperaturas en este ensayo, los resultados demostraron que no hay alteración del ADN del parásito en los insectos infectados experimentalmente en ninguna de las combinaciones realizadas (figura 2). Teniendo en cuenta que los resultados obtenidos con etanol al $70 \%$ a temperatura ambiente son iguales, por un lado, a los obtenidos con esta misma solución a $-20^{\circ} \mathrm{C}$ y $-80{ }^{\circ} \mathrm{C}$ y, por el otro, con los de las otras dos soluciones, etanol al $100 \%$ y con TE (buffer Tris-EDTA), en las tres temperaturas evaluadas. Por costos, facilidad de adquisición y manejo de las muestras en condiciones de campo, para estudios de incriminación de

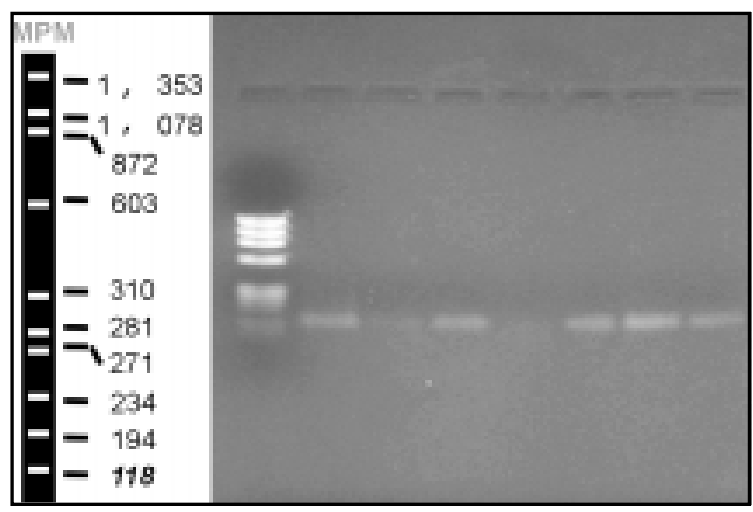

Figura 1. Resultados de las muestras en diferentes medios a temperatura ambiente. Carril 1: MPM; carril 2: control positivo, cultivo L. chagasi; carril 3: control negativo, intestino de un insecto alimentado con la mezcla infectiva; carril 4: intestino con flagelados en TE, con visualización de la banda; carril 5: muestra de un insecto expuesto al alimento infectivo sin visualización de la banda; carriles 6,7 y 8 : insectos completos sin disecar expuestos al alimento infectivo, almacenados respectivamente en TE, etanol al $70 \%$ y etanol $100 \%$ con visualización de la banda.

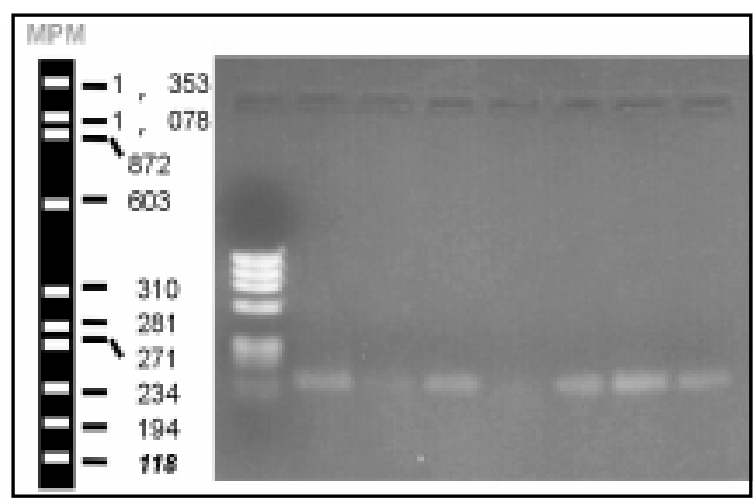

Figura 2. Resultados de las muestras en diferentes medios y temperaturas. Carril 1: MPM; carril 2: control positivo, cultivo L. chagasi; carril 3: control negativo, insecto de colonia en TE; carril 4: muestra de un insecto expuesto al alimento infectivo, almacenado en TE a $-70^{\circ} \mathrm{C}$, con visualización de la banda; carriles 5 y 7 : insectos completos sin disecar expuestos al alimento infectivo, almacenados en etanol al $70 \%$ a temperatura ambiente, con visualización de la banda; carriles 6 y 8 : intestino con flagelados en etanol al $100 \%$ a $\quad-20{ }^{\circ} \mathrm{C}$ con visualización de la banda.

vectores de leishmaniasis, se recomienda utilizar etanol al $70 \%$ a temperatura ambiente.

No obstante, se necesitan futuros estudios para llegar a la caracterización e identificación etiológica de los parásitos y vectores comprometidos en los ciclos de transmisión en áreas endémicas de leishmaniasis en el país, especialmente de leishmaniasis cutánea, ya que parásitos y vectores varían con la distribución geográfica de la enfermedad (32).

En conclusión, para estudios de incriminación vectorial, el etanol al $70 \%$ a temperatura ambiente es la forma de almacenamiento adecuada para insectos del género Lutzomyia. Además, estos resultados muestran la ventaja de incorporar la PCR para detectar flebótomos infectados en zonas endémicas de leishmaniasis visceral, como técnica de rutina.

\section{Agradecimientos}

A Magaly Sandoval del CINTROP-UIS, por su asesoría y colaboración; a Martha Ayala del Laboratorio de Parasitología del INS por el suministro del cultivo de Leishmania chagasi; a Víctor A. Olano del Laboratorio de Entomología por la lectura del documento. El estudio fue financiado por el Instituto Nacional de Salud de 
Colombia y los National Institutes of Health de Estados Unidos, a través de Yale University.

\section{Referencias}

1. De Azebedo A, Monteiro F, Cabello PH, De Souza NA, Rosa-Freitas MG, Rangel EF. Studies on populations of Lutzomyia longipalpis (Lutz \& Neiva, 1912) (Diptera: Psychodidae: Phlebotominae) in Brazil. Mem Inst Oswaldo Cruz 2000;95:305-22.

2. Arrivillaga JC, Rangel YN, Oviedo M, Feliciangeli MD. Correlated morphologic and genetic diversity among Lutzomyia longipalpis (Diptera: Psychodidae) collections in Venezuela. J Am Mos Control Assoc 2000; 16:1714.

3. Arrivillaga JC, Rangel YN, Oviedo M, Feliciangeli MD. Genetic divergence among Venezuelan populations of Lutzomyia longipalpis (Diptera: Psychodidae: Phlebotominae). J Med Entomol 2000;37:325-30.

4. Young DG, Duncan M. Guide to the identification and geographic distribution of Lutzomyia sand flies in Mexico, the West Indies, Central and South America (Diptera: Psychodidae). Mem Am Entomol Inst 1994;54: 1-881.

5. Travi BL, Vélez ID, Brutus L, Segura I, Jaramillo C, Montoya J. Lutzomyia evansi an alternate vector of Leishmania chagasi in a Colombian focus of visceral leishmaniasis. Trans R Soc Trop Med Hyg 1990;84:6767.

6. Zeledón R, Murrillo J, Gutiérrez H. Observaciones sobre la ecología de Lutzomyia longipalpis (Lutz \& Neiva, 1912) y posibilidades de existencia de leishmaniasis visceral en Costa Rica. Mem Inst Oswaldo Cruz 1984;79:455-9.

7. Feliciangeli MD, Rodriguez N, De Guglielmo Z, Rodriguez A. The re-emergence of American visceral leishmaniasis in an old focus in Venezuela. II. Vectors and parasites. Parasite 1999;6:113-20.

8. Killick-Kendrick R. Phlebotomine vectors of the leishmaniasis: a review. Med and Vet Entomol 1990;4:124.

9. Young DG, Morales A, Kreutzer RD, Alexander JB, Corredor A, Tesh RB. Isolations of Leishmania braziliensis from cryopreserved Colombian sand flies (Diptera: Psychodidae). J Med Entomol 1987;24:587-9.

10. Ferro C, Morrison AC, Torres M, Pardo R, Wilson ML, Tesh RB. Age structure, blood-feeding behavior, and Leishmania chagasi infection in Lutzomyia longipalpis (Diptera: Psychodidae) at an endemic focus of visceral leishmaniasis in Colombia. J Med Entomol 1995;32:618-29.

11. Uribe S, Porter C, Velez ID. Amplificación y obtención de secuencias de rRNA mitocondrial en Lutzomyia spp. (Diptera: Psychodidae) vectores de leishmaniasis. Rev Colombiana Entomol 1998;24:109-15.
12. Aransay AM, Scoulica E, Tselentis Y. Detection and identification of Leishmania DNA within naturally infected sand flies by seminested PCR on minicircle kinetoplastic DNA. Appl Environ Microbiol 2000;66:1933-8.

13. Uezato H, Hagiwara K, Hosokawa A, Maruno M, Nonaka S, Oshiro M, et al. Comparative studies of the detection rates of Leishmania parasites from formalin, ethanol-fixed, frozen human skin specimens by polymerase chain reaction and Southern blotting. J Dermatol 1998;25:623-31.

14. Rodríguez N, Guzmán B, Rodas A, Takiff H, Bloom BR, Convit J. Diagnosis of cutaneous leishmaniasis and species discrimination of parasites by PCR and hibridization. J Clin Microbiol 1994;32:2246-52.

15. Pirmez C, Trajano V, Paes-Oliveira M, Da-Cruz AM, Goncalves DA, Costa SC, et al. Use of PCR in diagnosis of human American tegumentary leishmaniasis in Rio de Janeiro, Brazil. J Clin Microbiol 1999;37:1819-23.

16. Romero GAS, Guerra MVF, Paes MG, Cupolillo E, Toaldo CB, Macêdo Vo, et al. Sensitivity of the polymerase chain reaction for the diagnosis of cutaneous leishmaniasis due to Leishmania (Viannia) guyanensis. Acta Trop 2001;79:225-9.

17. Barrios MA, Rodríguez N, Feliciangeli MD, Ulrich M, Telles S, Pinardi ME, et al. Coexistence of two species of Leishmania in the digestive tract of the vector Lutzomyia ovallesi. Am J Trop Med Hyg 1994;51:66975.

18. Rodríguez N, De Lima H, Rodríguez A, Brewster S, Barker DC. Genomic DNA repeat from Leishmania (Viannia) braziliensis (Venezuelan strain) containing simple repeats and mocrosatelites. Parasitology 1997; 115:349-58.

19. Mukherjee S, Hassan MQ, Ghosh A, Ghosh KN, Bhattacharya A, Adhya S. Short report: Leishmania DNA in Phlebotomus and Sergentomyia species during a kala-azar epidemic. Am J Trop Med Hyg 1997;57:4235.

20. Schriefer ME, Sacci JB, Wirtz RA, Azad AF. Detection of polymerase chain reaction-amplified malarial DNA in infected blood and individual mosquitoes. Exp Parasitol 1991;73:311-6.

21. Sandoval M, Gutiérrez R, Cárdenas R, Cabrera OL, Ferro C. Estandarización de la técnica de reacción en cadena de la polimerasa para estudios de infección natural de Lutzomyia (Diptera: Psychodidae). Biomédica 1999;19:122.

22. Killick-Kendrick R, Leaney AJ, Ready PD. The establishment, maintenance and productivity of a laboratory colony of Lutzomyia longipalpis (Diptera: Psychodidae). J Med Entomol 1977;13:429-40. 
23. Morrison AC, Ferro C, Morales A, Tesh R, Wilson ML. Dispersal of the sand fly Lutzomyia longipalpis (Diptera: Psychodidae) at an endemic focus of visceral leishmaniasis in Colombia. J Med Entomol 1993;30:427-35.

24. Oliveira-Neto MP, Pirmez MC, Fernandes O, Gonçalves-Costa SC, Silva de Souza SF, Grimaldi G Jr. Mucosal leishmaniasis ("espundia") responsive to low dose of n-methyl glucamine (Glucantime ${ }^{\circledR}$ ) in Rio de Janeiro, Brazil. Rev Inst Med trop S Paulo 2000; 42:321-5.

25. Miranda JC, Reis E, Gonçalves M, Reis M, Fernades O, Barral-Netto $\mathrm{M}$, et al. Combination of pinpointed phlebotomine capture and PCR for Leishmania improves detection of naturally infected Lutzomyia. Mem Inst Oswaldo Cruz 1997;92:504.

26. Rogers WO, Burnheim PF, Wirth DF. Detection of Leishmania within sand flies by kinetoplast DNA hibridization. Am J Trop Med Hyg 1988;39:434-9.

27. Lopez M, Inga R, Cangalaya M, Echevarría J, LlanoCuentas A, Orrego C, et al. Diagnosis of Leishmania using the polymerase chain reaction: a simplified procedure for field work. Am J Trop Med Hyg 1993;49:348-56
28. Feliciangeli MD, Rodríguez N, Bravo A, Arias F, Guzmán B. Vectors of cutaneous leishmaniasis in north-central Venezuela. Med Vet Entomol 1994;8:31724.

29. Noyes HA, Reyburn H, Bailey W, Smith D. A nestedPCR-based schizodeme method for identifying Leishmania kinetoplast minicircle classes directly from clinical samples and its application to the study of the epidemiology of Leishmania tropica in Pakistan. J clin Microbiol 1998;36:2877-81.

30. Rodríguez N, Aguilar CM, Barrios MA, Barker DC. Detection of Leishmania braziliensis in naturally infected individual sand flies by the polymerase chain reaction. Trans R Soc Trop Med Hyg 1999;93:47-9.

31. Isaza DM, Arboleda M, Restrepo M, McCann SHE, Barker DC. Validation of the polymerase chain reaction for the diagnosis of human cutaneous leishmaniasis in north-west Colombia. Trans R Soc Trop Med Hyg 2002; 96:165-8

32. Ferro C, Morales A. Flebótomos de ColXXombia: estudios realizados por el Laboratorio de Entomología 1965-1997. En: Toro G, Hernández CA, Raad J, editores. Instituto Nacional de Salud, 1917-1997: una historia, un compromiso. Bogotá, D.C.: Instituto Nacional de Salud; 1998. p.77-94. 